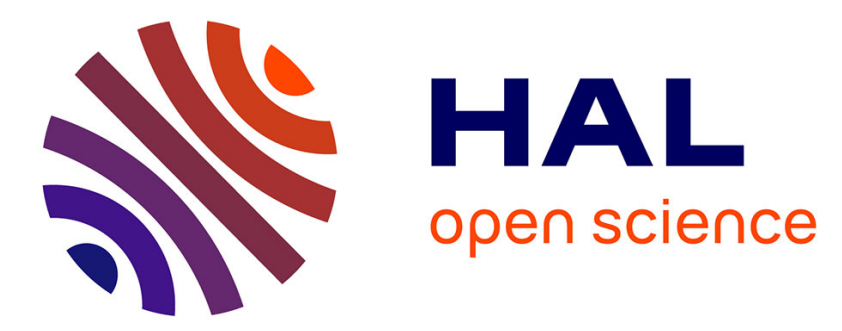

\title{
Echo Response of Faults in Transmission Lines: Models and Limitations to Fault Detection
}

\author{
Andréa Cozza, Lionel Pichon
}

\section{To cite this version:}

Andréa Cozza, Lionel Pichon. Echo Response of Faults in Transmission Lines: Models and Limitations to Fault Detection. IEEE Transactions on Microwave Theory and Techniques, 2016, 64 (12), pp.4155 - 4164. 10.1109/TMTT.2016.2608774 . hal-01352701

\section{HAL Id: hal-01352701 \\ https://hal-centralesupelec.archives-ouvertes.fr/hal-01352701}

Submitted on 9 Aug 2016

HAL is a multi-disciplinary open access archive for the deposit and dissemination of scientific research documents, whether they are published or not. The documents may come from teaching and research institutions in France or abroad, or from public or private research centers.
L'archive ouverte pluridisciplinaire HAL, est destinée au dépôt et à la diffusion de documents scientifiques de niveau recherche, publiés ou non, émanant des établissements d'enseignement et de recherche français ou étrangers, des laboratoires publics ou privés. 


\title{
Echo Response of Faults in Transmission Lines: Models and Limitations to Fault Detection
}

\author{
Andrea Cozza, Senior Member, IEEE, Lionel Pichon
}

\begin{abstract}
This paper introduces models of the time-domain echoes generated by faults in transmission lines excited by test signals, e.g., as in applications of time-domain reflectometry. Faults here considered include local modifications of the propagation characteristics of a transmission line. It is shown that the response of faults are strongly dispersive in nature, which implies that the peak of their echo is far from providing an accurate measure of the severity of the fault, as it heavily depends on the frequency content of the test signal, as well as on the length of the fault. It is argued that fault detection in transmission lines is an ill-posed problem that requires a priori knowledge on the fault itself. These results are important for applications of time-domain reflectometry methods, particularly for early-warning monitoring of potentially critical faults from their onset, since it is shown that echoes from faults tested at relatively low frequencies can lead to underestimate their actual severity.
\end{abstract}

Index Terms-Transmission lines, fault detection, soft faults, echo detection, time-domain reflectometry.

\section{INTRODUCTION}

Transmission lines are subject to unwanted modifications, such as partial cuts in their coating and shielding, changing distances between its conductors, filling medium, etc. Modifications of this kind are seldom a critical issue, though they can affect the integrity of signal/energy transmission infrastructures. More importantly, the repetitive action of external factors (static forces, vibrations, thermal expansions, corrosive products, etc.) can eventually lead to a permanent and irreversible modification in the geometry and/or materials in a transmission line. Typically, modifications of this kind occur over very short portions of a line, in the millimeter range.

Since such modifications can affect the nominal behavior of a transmission line, it is common to refer to them as faults. For clear reasons, the most important modifications are short and open circuits: these are usually called hard faults and can electrically severe a line into two separate portions. But their onset can be related to less critical faults, sometimes referred to as soft faults, which can eventually develop into hard faults. It is important to be capable of detecting faults before they reach a critical state, when they still act as weak perturbations in the nominal behavior of a line.

While general faults can take a number of shapes (partial cuts, crushed conductors, etc.), they all share the same structure, depicted in Fig. 1: the nominal characteristic impedance $Z_{o}$ of a line is locally modified to a value $Z_{F}$, over a section of length $w$. The transition region between the nominal and

A. Cozza and L. Pichon are with PIEM, Group of Electrical Engineering Paris (GeePs), UMR 8507 CentraleSupelec, Univ Paris-Sud, UPMC, CNRS, 11 rue Joliot-Curie, 91192 Gif-sur-Yvette, France.

Contact e-mail: andrea.cozzalieee.org

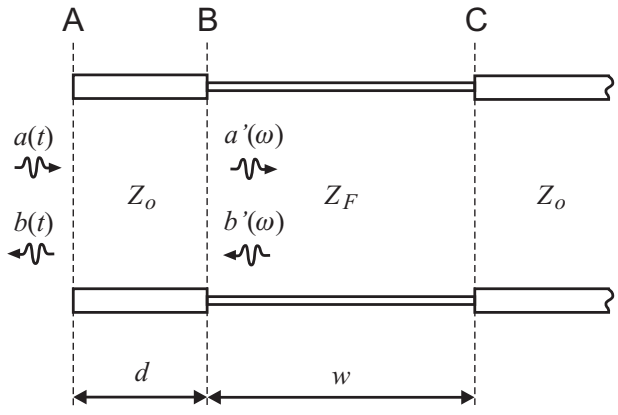

Fig. 1. Double impedance-step representation of a local fault in a transmission line of characteristic impedance $Z_{o}$, and relevant quantities for the derivation of the response $\Gamma_{F}(\omega)$ of a fault of impedance $Z_{F}$.

modified lines is assumed to be much shorter than $w$, and will thus be neglected as a second-order contribution. See [1] for a review of typical faults in two-wire transmission lines.

The most widely used approach for detecting faults in transmission lines is the extended group of time-domain reflectometry (TDR) techniques. In a general manner, their aim is to detect the presence (and ideally the position and severity) of a discontinuity in a transmission line, by submitting it to a test signal $a(t)$ through an electrical port, while monitoring the reflected signal $b(t)$ [2]-[4].

Assuming a reflected signal proportional to the test signal, one should ideally be capable of assessing the severity of the fault, e.g., expressed through its reflection coefficient at the fault position

$$
\Gamma_{o}=\frac{Z_{F}-Z_{o}}{Z_{F}+Z_{o}}
$$

which, for weakly lossy lines is fundamentally real-valued. $\Gamma_{o}$ thus provides an effective measure of the deviation from the nominal impedance of the line. $\Gamma_{o}$ as described in (1) should not be thought of as an input reflection coefficient measured at one end of a line, but as the reflection introduced by the fault at its position along the line under test.

This standard interpretation of echoes from single-step discontinuities is routinely applied to any TDR application: not only in the actual case of loads at the end of a line or the case of a open or short circuit along a line, where it is justified, but it is also extended to other configurations, such as the case of local modifications in the propagation parameters of a transmission line [5]-[7], the general kind of fault discussed in this paper.

Previous investigations into the special case of soft faults were presented in [1]. While correctly modeling a fault as a two-step discontinuity, and acknowledging the existence 
of a double reflection as the physical mechanism behind weak echoes, it falls short of deriving models describing the time-domain response, or echoes, generated by faults when submitted to test signals. In a more general way, it can be noticed that the major motivation in most papers on TDR techniques is increasing the contrast between fault echoes and other unrelated signals, with little attention paid to eventual differences between the shape of the test and echo signals. The use of narrow-band test signals makes things worse, as they do not allow to easily infer differences between test and echo signals. The end of sec. III provides details about this conclusion.

Faults in transmission lines are characterized by three parameters: 1) the single-step reflection coefficient $\Gamma_{o}$, defined as in $(1), 2)$ the fault extension $w$ and 3 ) the propagation speed $c$ along the faulty section. All of them take part in the definition of the response of a fault to a test signal.

In fault detection one mostly looks for the position and severity $\left|\Gamma_{o}\right|$ of the fault, which is assumed (sometimes implicitly) throughout available literature as being the proportional coefficient between test signals and echoes [7], [8], thus directly accessible.

It is the aim of this paper to prove that this assumption is incorrect and that faults in transmission lines are characterized by echo responses that are not simply proportional to the test signal, but are rather more closely related to its first time derivative. Sec. II introduces models of the response of a fault to test signals, for different special cases. The practical implications of these results are discussed in sec. III, with a particular attention to potential errors and ambiguities in the interpretation of TDR results, while a numerical validation is presented in sec. IV. Two main results are demonstrated in this paper: the impossibility of assessing the severity of a fault without prior information or assumptions on its physical extension and the very high risk of underestimating the severity of a fault if expecting it to be related to the peak amplitude of the echoes it produces. Alternative procedures exist, at least for soft-fault detection, that are not based on echo processing but rather on subspace processing [9], [10], implemented in the frequency-domain, where fault severity is not based on the amplitude of the echoes they procude.

\section{FAULT MODELS}

We are here interested in modelling the interaction of an impinging signal $a(t)$ with a fault described as in Fig. 1. Wave propagation will be assumed to be dominated by a TEM or quasi-TEM mode, as found in the majority of cables used in practical scenarios. Since test signals are usually limited to the VHF-UHF bandwidths, higher-order modes can be neglected. Edge effects will also be neglected, assuming propagation to be the dominant physical phenomenon. These include direct capacitive coupling between the two edges of a faulty section and could thus have an impact at low frequencies for a very short fault, breaking the translation invariance underlying transmission-line theory of uniform lines.
In order to simplify our derivation, we will work in the frequency domain, where the Fourier-spectrum of the reflected signal can be expressed as

$$
b(\omega)=\Gamma_{F}(\omega) a(\omega),
$$

with $\Gamma_{F}(\omega)$ the reflectivity of the fault, as measured from the left of the reference plane $A$. The propagation over the section A-B is described by means of forward and backward propagating power waves $a(\omega)$ and $b(\omega)$, respectively, while over the fault section it will be described by another set of such waves, noted as primed quantities in Fig. 1. In order to derive $\Gamma_{F}(\omega)$, it suffices to impose the continuity of voltage and current over the plane B.

To this effect, we need to recall the relationships between the voltage $V(\omega)$, the current $I(\omega)$ and power waves along a uniform transmission line of characteristic impedance $Z_{c}[11]$, [12]

$$
\begin{aligned}
& a(\omega)=\left[V(\omega)+Z_{c} I(\omega)\right] / 2 \sqrt{Z_{c}} \\
& b(\omega)=\left[V(\omega)-Z_{c} I(\omega)\right] / 2 \sqrt{Z_{c}},
\end{aligned}
$$

where the characteristic impedance will be assumed to be frequency independent, as expected for weakly dispersive structures. For the case in Fig. 1, imposing the continuity of the voltage and current across the reference plane $A$ results in

$$
\begin{aligned}
{\left[a(\omega) \mathrm{e}^{-\mathrm{j} k_{o} d}+b(\omega) \mathrm{e}^{+\mathrm{j} k_{o} d}\right] \sqrt{Z_{o}} } & =\left[a^{\prime}(\omega)+b^{\prime}(\omega)\right] \sqrt{Z_{F}} \\
{\left[a(\omega) \mathrm{e}^{-\mathrm{j} k_{o} d}-b(\omega) \mathrm{e}^{+\mathrm{j} k_{o} d}\right] / \sqrt{Z_{o}} } & =\left[a^{\prime}(\omega)-b^{\prime}(\omega)\right] / \sqrt{Z_{F}},
\end{aligned}
$$

where $k_{o}=\omega / c_{o}$ is the propagation constant for the nominal line, with $c_{O}$ the associated propagation speed. These two equations can be combined together, yielding

$$
\begin{aligned}
& 2 a(\omega) \mathrm{e}^{-\mathrm{j} k_{o} d}=a^{\prime}(\omega) \beta_{+}+b^{\prime}(\omega) \beta_{-} \\
& 2 b(\omega) \mathrm{e}^{+\mathrm{j} k_{o} d}=a^{\prime}(\omega) \beta_{-}+b^{\prime}(\omega) \beta_{+},
\end{aligned}
$$

with $\beta_{ \pm}=\zeta \pm 1 / \zeta$ and $\zeta=\sqrt{Z_{F} / Z_{o}}$. Since

$$
b^{\prime}(\omega)=-\Gamma_{o} a^{\prime}(\omega) \mathrm{e}^{-\mathrm{j} 2 k w},
$$

with $k=\omega / c$ the propagation constant for the faulty section and $c$ the associated propagation speed, recalling (2), the reflectivity of the fault measured from section A can be written as

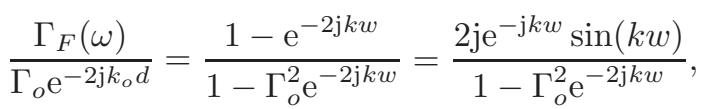

where $\Gamma_{o} \mathrm{e}^{-2 \mathrm{j} k_{o} d}$ is the reflection generated by the first discontinuity at the beginning of the faulty section. We will systematically study the ratio $\Gamma_{F} / \Gamma_{o}$ throughout the paper, as it provides a direct measure of the differences between the actual reflection coefficient $\Gamma_{F}$ as observed from a testing port, with respect to the single-step reflection coefficient $\Gamma_{o}$ which, though not directly accessible, quantifies the severity of a fault as a modification in the characteristic impedance of the line.

Eq. (7) asserts that the double-step discontinuity found in local faults does not react to test signals producing echoes proportional to $\Gamma_{o}$, apart in presence of hard faults $\left(\Gamma_{o} \rightarrow \pm 1\right)$. Far more important are the practical implications of (7), in 

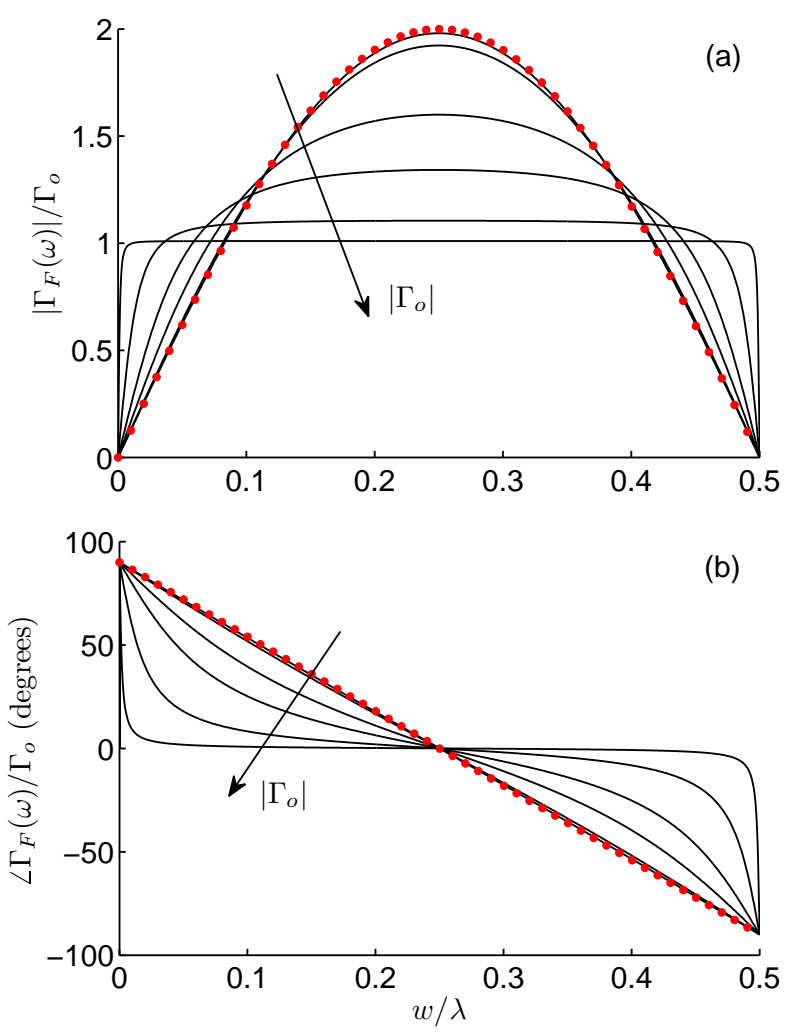

Fig. 2. Amplitude and phase-shift angle for the echo response $\Gamma_{F}(\omega)$ predicted by (7), for six values of $\left|\Gamma_{o}\right|$, namely $0.1,0.2,0.5,0.7,0.9$ and 0.99 , in increasing order according to the direction of the arrows in the graphics. The red dots represent the soft-fault (weak perturbation) approximation (8).

particular understanding under what conditions the severity of a fault (i.e., $\Gamma_{o}$ ) can be accurately estimated. A special attention will be paid to the time-domain responses of soft faults, which are sometimes naively regarded as producing scaled-down echoes similar to those of hard faults. Our results prove that apart for the case of hard faults, assessing a fault severity from TDR echoes is an ill-posed problem.

In the following sections approximations of (7) derived for special configurations will be presented. The distance $d$ will be assumed equal to zero for the sake of simplicity. Due to the periodicity of $\Gamma_{F}(\omega)$, we will limit our analysis to the first period. In fact, only the lower frequency region of this first period is actually of interest as long as $w \lesssim \lambda$, with $\lambda$ the shortest guided wavelength associated to the test signal.

\section{A. Soft faults}

In the case the impedance discontinuity can be regarded as a weak perturbation of the nominal one, i.e., with $Z_{F} \simeq Z_{o}$ or $\left|\Gamma_{o}\right| \ll 1$, (7) can be expressed as

$$
\frac{\Gamma_{F}(\omega)}{\Gamma_{o}}=2 \mathrm{je}^{-\mathrm{j} k w} \sin (k w)+\mathcal{O}\left(\Gamma_{o}^{2}\right) .
$$

This result is compared with the general expression (7) in Fig. 2, where (8) appears to be a good approximation as long as $\left|\Gamma_{o}\right|<0.2$, i.e., for $Z_{F} / Z_{o} \in[0.67,1.5]$. This relatively large range of deviations from the nominal impedance implies that soft faults should not necessarily be expected to correspond to very weak modifications, as confirmed in the results presented in sec. IV.

Approximation (8) could also be derived directly by applying to the line in Fig. 1 the small-reflection approximation described in [12]: the testing wave is first reflected at section $\mathrm{B}$, with a constant reflectivity $\Gamma_{o}$, while practically heading unmodified towards section $\mathrm{C}$, where it would undergo the same phenomenon, but this time with a reflection coefficient $-\Gamma_{o}$. Multiple interactions along the faulty section are neglected, as reasonable for a vanishingly low $\Gamma_{o}$. Depending on the fault length $w$, the two echoes can therefore partially cancel out, leading to a weak overall echo. This mechanism was already highlighted in [1].

While (8) is valid over a wide frequency range, its practical implications are not easily apparent. Moreover, the tendency to associate weak echoes to soft faults is demonstrated to be incorrect in sec. IV. More general and useful expressions are proposed in the next two sections.

\section{B. Electrically-short faults}

The results in Fig. 2 show that as long as $w / \lambda \lesssim 1 / 4$ the response $\Gamma_{F}(\omega)$ resembles that of a high-pass filter, but for the case of soft faults. This idea can be put into equations by looking for an approximation of the kind

$$
\frac{\Gamma_{F}(\omega)}{\Gamma_{o}} \simeq \frac{A \mathrm{j} \omega}{\mathrm{j} \omega+p},
$$

where $p$ is a real-valued pole and $A$ a constant. These two quantities can be found by computing the Padé approximant of (7), for the case of a first degree numerator and denominator. Padé approximants are the best approximation of an original function at a given point, since it ensures that all the derivatives of the original and approximated functions coincide at a reference point [13], here chosen to be $\omega=0$.

The result of this procedure is

$$
\begin{aligned}
A & =\frac{2}{1+\Gamma_{o}^{2}} \\
p & =\frac{1-\Gamma_{o}^{2}}{1+\Gamma_{o}^{2}} \frac{c}{w} .
\end{aligned}
$$

The exact result (7) is compared with the zero-pole approximation (9) in Fig. 3, for different values of $\Gamma_{o}$. As expected, the approximation works well in the lower frequency range, where the fault is electrically short. In order to verify this condition, it is convenient to define the characteristic frequency of the fault, i.e., the frequency at which the multiple reflections at the two ends of the fault are in phase and would lead to a resonance, as

$$
f_{c} \doteq \frac{c}{w} \simeq \frac{30}{\sqrt{\epsilon_{\mathrm{e}}} w_{\mathrm{cm}}} \mathrm{GHz},
$$

here expressed in $\mathrm{GHz}$ for a fault extension measured in centimeters; $\epsilon_{\mathrm{e}}$ is the effective relative dielectric constant of the faulty section. Since $w / \lambda=f / f_{c}$, (11) shows that the assumption of an electrically short fault holds in practical situations where a fault is typically shorter than a centimeter and the test signals seldom reach the $\mathrm{GHz}$ range. 

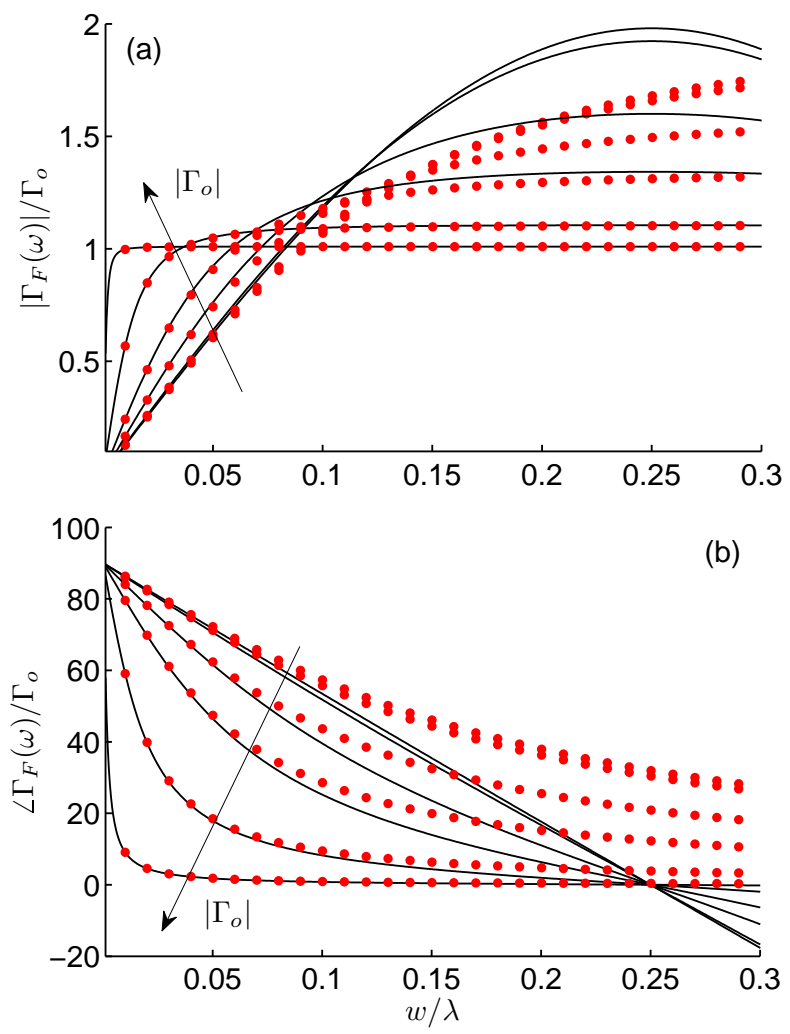

Fig. 3. Comparison of the exact (black solid lines) and zero-pole (red dots) models for the fault echo response $\Gamma_{F}(\omega)$. Six different values of $\Gamma_{o}$ are shown, 0.1, 0.2, 0.5, 0.7, 0.9 and 0.99, in increasing order according to the direction of the arrows in the graphics.

These results show that the effects of the presence of the pole are more heavily felt as soon as $\Gamma_{o}$ increases, even at relatively low frequencies. While (10) states that $p$ will move at higher frequencies for soft faults, for harder faults it will appear well before. The accuracy of the approximation for soft faults is not as good since it is dominated by delay terms, which cannot be well approximated by means of a finite number of poles, as well known from control theory [14]. Yet, its accuracy strongly varies with the frequency range spanned by the test signals used for TDR fault detection. In particular, in the lower-frequency range the approximation is rather good, as shown later.

It is therefore useful to regard $f_{o}=p / 2 \pi$ as a critical frequency of the fault, since it determines the nature of its echoes, as discussed in sec. III.

The main advantage of (9) with respect to (7) is that the former can be transformed into a simple time-domain expression, thus providing the opportunity to understand how a fault responds to test signals. The result of this operation is

$$
\Gamma_{F}(t) \simeq \frac{2 \Gamma_{o}}{1+\Gamma_{o}^{2}}\left[\delta(t)-p u(t) \mathrm{e}^{-p t}\right]
$$

where $\delta(t)$ is Dirac's delta distribution and $u(t)$ is Heaviside's unit-step function.

The main effect of the pole is observable in the exponential term in (12): its practical impact will be discussed in sec. III. It is already clear from (12) that the echo resulting from a fault
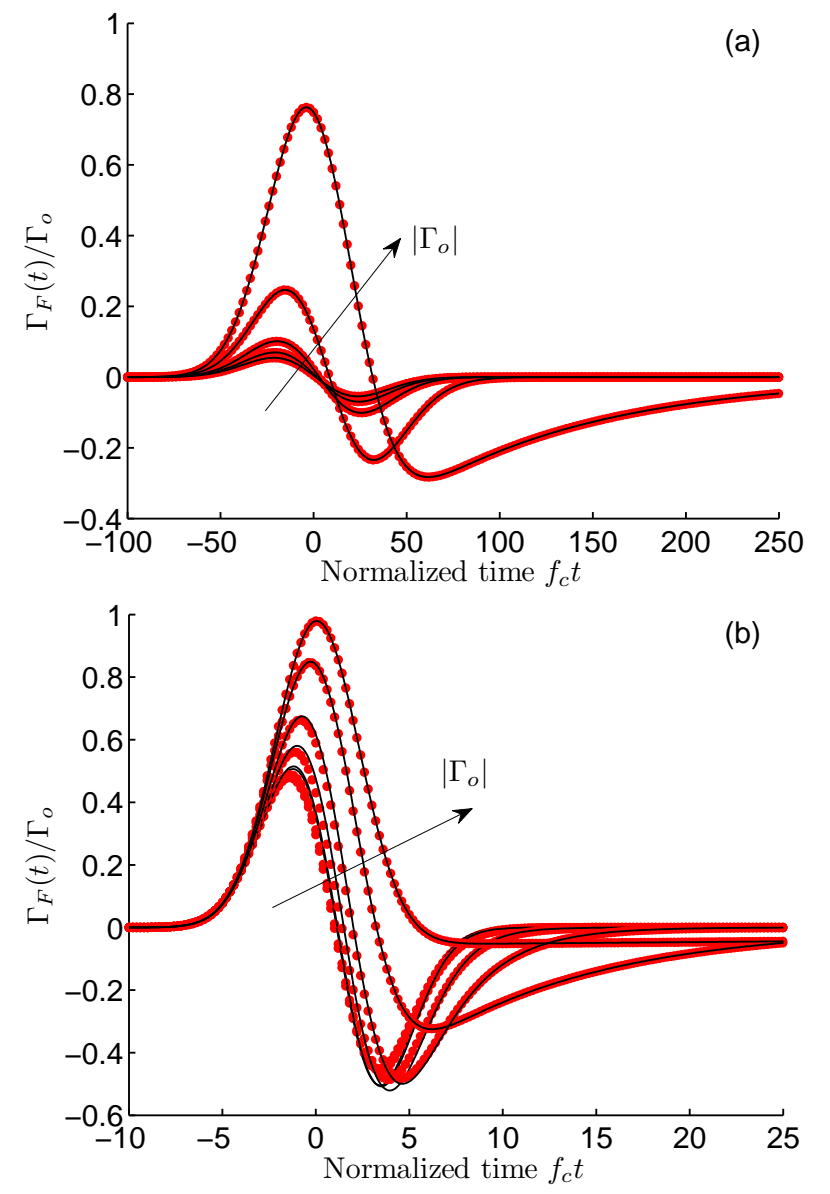

Fig. 4. Normalized time-domain echoes for an electrically-short fault, expected for a baseband Gaussian test signal with (a) $B_{o} / f_{c}=0.01$ and (b) $B_{o} / f_{c}=0.1$. The different curves correspond to increasing values of $\Gamma_{o}$ equal to $0.1,0.2,0.5,0.7,0.9$ and 0.99 , according to the direction of the arrows. Exact results (black solid lines) and approximations from the zero-pole model (red dots) are shown.

is certainly not simply proportional to the test signal, due to this additional exponential term, which makes the response dispersive. In the case of hard faults (12) simplifies into

$$
\lim _{\left|\Gamma_{o}\right| \rightarrow 1} \Gamma_{F}(t)=\Gamma_{o} \delta(t)
$$

as expected for a line terminated by a short or open circuit. Only in this case, the echo follows the original shape of the test signal.

Examples of the echoes expected under the electrically-short fault condition are shown in Fig. 4, obtained for a baseband unit Gaussian test signal

$$
\begin{aligned}
a(t) & =\mathrm{e}^{-\left(t / T_{o}\right)^{2} / 2} \\
a(\omega) & =\sqrt{2 \pi} T_{o} \mathrm{e}^{-\left(\omega / 2 \pi B_{o}\right)^{2} / 2},
\end{aligned}
$$

where $T_{o}$ is a time-scale constant and $B_{o}=1 / 2 \pi T_{o}$. The results in Fig. 4 refer to a set of faults of same length, for several values of $\Gamma_{o}$. Two different frequency bandwidths where considered for the test signal, namely $B_{o} / f_{c}$ equal to 0.01 and 0.1 , with $f_{c}$ the characteristic frequency of the fault defined in (11). While in both cases the faults can be 


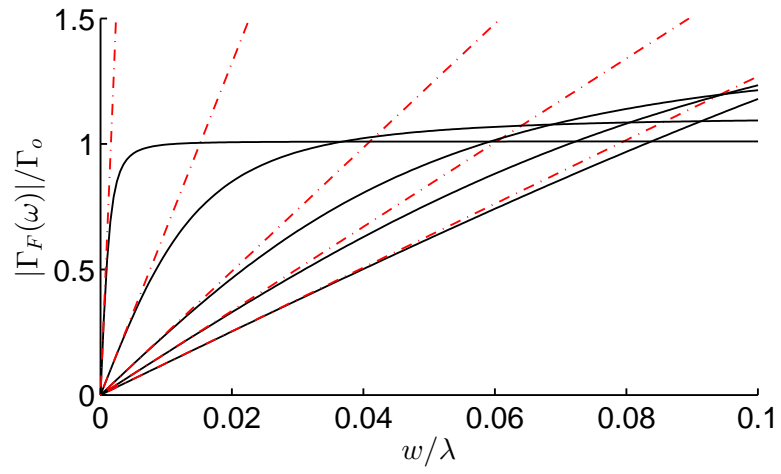

Fig. 5. Comparison between the exact model (7), solid lines, and the low-frequency approximation (16), red dash-dot lines, for the values $\Gamma_{o}=$ $\{0.3,0.5,0.7,0.9,0.99\}$.

considered as electrically short, the echoes they produce can be quite different in shape and amplitude.

If fault echoes were directly proportional to the reflection coefficient $\Gamma_{o}$, then all the normalized echoes in Fig. 4 should attain a peak value equal to 1 at $t=0$. In fact, this condition is almost met only in the case of a hard fault with $\Gamma_{o}=0.99$, for the case of a very wide bandwidth, $B_{o} / f_{c}=0.1$. Indeed, while still within the short-fault assumption, it already implies a need for ultra-wide band signals, since taking as an example the case of a $1-\mathrm{cm}$ long fault in a line with $\epsilon_{\mathrm{e}}=1$, (11) requires that $B_{o}=3 \mathrm{GHz}$, which is not a usual choice for a test signal.

In all the other cases the peak reflection is lower than expected for the single-step paradigm. Moreover, as $\Gamma_{o}$ decreases, the tail occurring in the late-time response of the echoes gives way to an odd-symmetry echo with lower amplitude. This transition was linked to the partial cancellation of echoes discussed in sec. II-A. The echoes now closely resemble the first time derivative of the test signal (see sec. II-C).

As soon as the bandwidth of the test signal is reduced, this trend becomes more pronounced, with echoes much weaker than expected from $\left|\Gamma_{o}\right|$. As discussed in sec. III, the risk of underestimating the actual severity of the fault is very likely, if the current use of the amplitude of echoes as a measure of the fault severity is maintained.

In all the results presented in Fig. 4 the comparison between the exact solution (7) and the short-fault approximation (9) is in good agreement, with some minor differences in the case of soft faults tested over a wide bandwidth, seen in Fig. 4(b).

\section{Low-frequency response : derivative approximation}

In practice, for test signals with a frequency content limited to frequencies somewhat smaller than $f_{o}$, or

$$
\frac{w}{\lambda}=\frac{f}{f_{c}} \lesssim \frac{f_{o}}{f_{c}}=\frac{1}{2 \pi} \frac{1-\Gamma_{o}^{2}}{1+\Gamma_{o}^{2}}
$$

(9) reduces to

$$
\frac{\Gamma_{F}(\omega)}{\Gamma_{o}} \simeq \frac{2 w / c}{1-\Gamma_{o}^{2}} \mathrm{j} \omega
$$

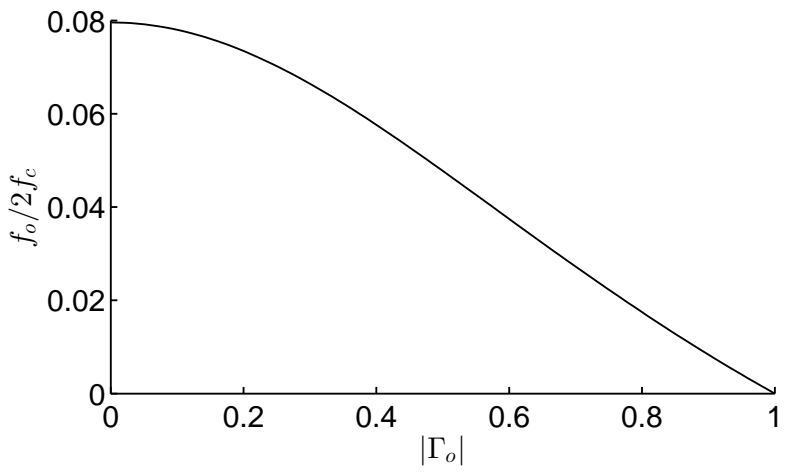

Fig. 6. Maximum normalized frequency $f / f_{c}$ for which the derivative approximation holds, as a function of the fault severity $\left|\Gamma_{o}\right|$, evaluated taking a safety factor 2 below the frequency $f_{o} / f_{c}$ in the condition (15).

i.e., to a derivative response, with an echo

$$
b(t) \simeq \frac{\Gamma_{o}}{1-\Gamma_{o}^{2}} \frac{2}{f_{c}} \dot{a}(t) .
$$

This approximation is expected to hold as long as $f / f_{c}$ is smaller than the value shown in Fig. 6, as required by (15), taking a safety factor equal to 2 .

Fig. 5 shows some comparisons of this approximation with the exact result (7), in the frequency domain. Among the results shown in Fig. 4, those satisfying the condition (15) do indeed yield a derivative echo, with a peak reflection much smaller than $\left|\Gamma_{o}\right|$ : this is the case in Fig. 4(a), for all configurations with $\left|\Gamma_{o}\right| \leqslant 0.9$. See next section for the practical implications related to this result.

\section{CONSEQUENCES ON THE INTERPRETATION OF ECHOES FOR FAULT DETECTION}

The results derived in the previous section are of practical importance, since they provide a better understanding of the conditions that lead a fault to respond in a seemingly different manner depending on the test signal. This claim can be better understood by taking the case of the baseband unit Gaussian test signal defined in (14). Under the low-frequency approximation (17), the peak value reached by the echo is equal to

$$
\max _{t}|b(t)|=\frac{\left|\Gamma_{o}\right|}{1-\Gamma_{o}^{2}} \frac{B_{o}}{f_{c}} \frac{4 \pi}{\sqrt{\mathrm{e}}} .
$$

The implications of this result are twofold: 1) the peak reflection should not be interpreted as a measure of the fault severity $\left.\left|\Gamma_{o}\right| ; 2\right)\left|\Gamma_{o}\right|$ could be assessed from (18) only if the characteristic frequency $f_{c}$ were known beforehand, which requires having access to the fault extension and to the propagation speed along the faulty section. While the order of magnitude of the propagation speed can be approximated with the nominal value expected for the original transmission line, the fault extension can vary wildly. In other words, the only quantity that can be properly assessed is $\Gamma_{o} / f_{c}$

$$
\frac{\left|\Gamma_{o}\right|}{f_{c}} \simeq \frac{\sqrt{\mathrm{e}}}{4 \pi B_{o}} \max _{t}|b(t)|,
$$


under the approximation $\left|\Gamma_{o}\right| \lesssim 1$. In fact, since $f_{c}$ is unknown, (18) cannot be solved for $\left|\Gamma_{o}\right|$ exactly, so that it is necessary to neglect the $1-\Gamma_{o}^{2}$ term in the denominator.

Moreover, the echo reaches its peak value not at an instant depending only on the position of the fault, but also on the shape of the test signal. As a matter of fact, (17) implies that

$$
\arg \max |b(t)|= \pm T_{o},
$$

here the inflexion points of the test signal, so that interpreting the echo of a fault as if it were proportional to the test signal leads to a systematic error about its position, since for a fault centered over the position $d$, one would come to the conclusion that the actual position is rather $d \pm T_{o} c_{o}$. For $B_{o}=30 \mathrm{MHz}$, the apparent fault position would be biased by about $1.6 \mathrm{~m}$, if $\sqrt{\epsilon_{e}}=1$. This bias only depends on the test signal.

Another source of ambiguity are the double-peak echoes resulting from pulsed test signals. Again, the standard interpretation of such echoes would lead to inferring the presence of two close faults. While we already recalled that for soft faults the echo can be regarded as such, (20) clarifies that this interpretation could be misleading. Since most of the time faults are tested over frequencies for which their electrical length is negligible, the double reflection generated by a generic fault would not translate into an identifiable double echo, but rather in the derivative of the test signal, since for short faults echoes are proportional to the derivative of the test signal, as discussed in sec. II-B.

The effect of an increasing bandwidth on the fault echoes are illustrated by the results in Fig. 4. The most striking implication is that for faults of the same severity, testing them over a narrower frequency bandwidth yields a weaker echo, even for $\Gamma_{o}$ as high as 0.9. It is therefore possible to dismiss a fault as not worth of attention, even though the line is already deeply modified, a direct consequence of the frequency-dispersive nature of a fault response. Realistic examples supporting this conclusion are presented in the next section. A more general implication is that a given fault can present different responses depending on the bandwidth of the test signal, producing echoes that can pass from derivative to proportional. A changing response is clearly a source of ambiguity in the interpretation of the nature of a fault.

In case the detection of an echo required exceeding a threshold voltage $v_{\text {th }}$, e.g., in connection to the noise background at the test port, then such a condition would translate into

$$
\frac{B_{o}}{v_{\mathrm{th}}}>\frac{\sqrt{2 \mathrm{e}}}{4 \pi} \frac{f_{c}}{\Gamma_{o} Z_{o}},
$$

for a Gaussian test signal. For the special case of $Z_{o}=50 \Omega$, (21) becomes

$$
\frac{B_{o}}{v_{\mathrm{th}}}>\frac{0.785}{\Gamma_{o} \sqrt{\epsilon_{e}} w_{\mathrm{cm}}} \quad \mathrm{GHz} / \mathrm{V},
$$

where $\epsilon_{e}$ is the effective dielectric constant of the line.

Hence, the proper detection of faults in transmission lines is more likely if using wideband test signals, unless the length of the fault is not negligible, or if it is very severe or if the signalto-noise ratio is very high, i.e., for a low detection threshold.
Finally, a simple way of making sure that an echo is caused by a fault would be to submit it to test signals of increasing bandwidth. As the peak reflection increases with the bandwidth, the echo could be pointed out as coming from a fault and not a single-step discontinuity: reflections at line junctions and loads would not change in their peak intensity.

While the derivative approximation does not allow retrieving at the same time $\Gamma_{o}$ and $f_{c}$, the structure of the zero-pole model (9) indicates that it should be possible do so, by fitting the parameters in (12) to the fault echo. To this end, it would be necessary to use test signals with a bandwidth extending beyond the critical frequency $f_{o}$. In practice, this option is hardly viable, since it would require very wide bandwidths not likely to be compatible with electronic systems connected to the line under test (see Sec. IV).

As a further example of currently used TDR test signals, the case of a signal $a_{e}(t)$ modulating a carrier at the frequency $f_{t}$ would imply

$$
\begin{aligned}
\dot{a}(t) & =\frac{\mathrm{d}}{\mathrm{d} t}\left\{a_{e}(t) \sin \left(2 \pi f_{t} t\right)\right\} \\
& =\dot{a}_{e}(t) \sin \left(2 \pi f_{t} t\right)+a_{e}(t) 2 \pi f_{t} \cos \left(2 \pi f_{t} t\right),
\end{aligned}
$$

which, for a narrow-band signal yields

$$
\dot{a}(t) \simeq 2 \pi f_{t} a_{e}(t) \cos \left(2 \pi f_{t} t\right)=2 \pi f_{t} a\left(t+1 / 4 f_{t}\right) .
$$

Hence, (17) would result into

$$
b(t) \simeq \frac{4 \pi \Gamma_{o}}{1-\Gamma_{o}^{2}} \frac{f_{t}}{f_{c}} a\left(t+1 / 4 f_{t}\right) .
$$

As for the case of the Gaussian test signal, the ratio between the echo peak amplitude and that of the test signal is not a direct measure of the fault severity, but is strongly dependent on the characteristics of the test signal, in particular the carrier frequency in this case. The additional delay associated to the fault echo is also dependent on the test signal and acts as a systematic error in the estimation of the fault position. The fact that for this kind of test signals the echo is practically proportional to the test signal comes with the risk of concluding that the single-step paradigm is accurate. The reason why the response of faults is often assumed as proportional likely lies there. Clearly, (25) shows that such an interpretation is not correct, as the intensity of the reflection depends on the frequency $f_{t}$ at which the line is tested. Moreover, the signature of a fault is again an echo intensity increasing with the frequency of the test signal: this property should help in the identification of line faults against reflections at junctions and loads, which have a proportional response, thus not dependent on the chosen test signal.

In the case of a unitary test signal, i.e., with $\max _{t}|a(t)|=1$, then the peak reflection from the fault echo allows assessing

$$
\frac{\left|\Gamma_{o}\right|}{f_{c}} \simeq \frac{\max _{t}|b(t)|}{4 \pi f_{t}},
$$

as similarly found in (19) for a baseband test signal, but in this case the intensity of the fault echo increases with the frequency of the carrier of the test signal.

In practice, one of the hardest issues in fault detection is to be able to discriminate reflections caused by harmless 
discontinuities (e.g., water drops along a line) from actual degradations [1]. Our models show the likely reason for this issue: the peak of fault echoes is proportional to $\left|\Gamma_{o}\right| / f_{c}$, thus also to $\left|\Gamma_{o}\right| w$. Hence, echoes of the same intensity can be generated by short faults of relatively high severity or longer ones of weaker severity. This ambiguity is inevitable and is confirmed in the numerical analysis presented in the next section.

\section{NUMERICAL ANALYSIS}

In this section we check the accuracy of the models introduced so far. Reference data are generated by means of numerical simulations. There are several reasons for choosing a numerical validation rather than an experimental one. First, it is necessary to know the characteristic impedance of the faulty section. In practice, given a faulty line, there is no simple way of de-embedding it from measurements, as claimed in this paper. Second, while removing a portion of a coaxial cable is not difficult, to ensure that it is done in a controlled and reproducible way is far less simple, since cables typically present transversal dimensions of a few millimeters, while mixing hard and soft materials, thus difficult to cut in a controlled way. Finally, it is important to test configurations as divers as possible, involving structures that are not easily reproduced in a laboratory setting.

The models derived in sec. II were validated by means of numerical simulations of coaxial (sec. IV-A) and two-wire transmission lines (sec. IV-B). The simulations were carried out with CST's Microwave Studio, over the frequency range from DC up to $12 \mathrm{GHz}$. We had to push the simulation to such high frequencies in order to confirm the dispersive response of faults. The wavelength at $12 \mathrm{GHz}$ is $2.5 / \sqrt{\epsilon_{e}} \mathrm{~cm}$, with $\sqrt{\epsilon_{e}}$ the effective refractive index of the dielectric materials in the line. Typical values of $\sqrt{\epsilon_{e}}$ are below 1.5 , so that the crosssection of the two-wire configuration tested in this section can be arguably regarded as still electrically small. Hence, the models derived in the previous sections can be expected to hold even at such high frequencies, since the conditions for assuming a dominant TEM-like propagation mode are met. The main issue in going to such high frequencies is the need to take into account propagation losses due to dissipation along the lines. We have chosen to neglect losses, for two reasons. First, losses can be accommodated into the proposed models, just by considering a complex propagation constant. Taking them into account would then only complicate the analysis, as it would introduce a further parameter to study. Second, test signals are typically designed well below the $\mathrm{GHz}$ range, where propagation losses are required to be negligible, as otherwise test signals interacting with faults and going back to the test port could be altered.

Several configurations of faults are presented in this section. For all of them, the numerical setup follows the structure in Fig. 1, with a nominal transmission line and a faulty portion of length $w$. For each configuration the characteristic impedance of the nominal and faulty sections were computed, as well as the propagation speed. The severity of the fault is assessed by computing the single-step reflection coefficient $\Gamma_{o}$, as defined in (1).

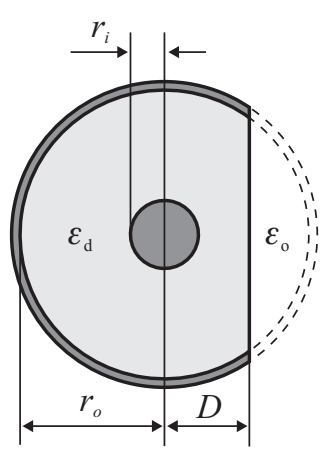

(a)

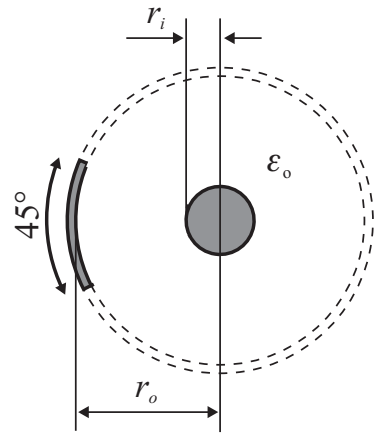

(b)
Fig. 7. Transversal cut of the coaxial line used in the numerical validation, with an internal conductor of radius $r_{i}=0.5 \mathrm{~mm}$, external conductor of radius $r_{o}=3 \mathrm{~mm}$, a dielectric with relative permittivity $\epsilon_{d}=2.1$ (polytetrafluoroethylene, PTFE). The modified line is (a) partially cut along a distance $D$ from its axis or (b) a simplified pigtail connection, where the external conductor is reduced to an angular region of 45 degrees, with the dielectric totally removed.

The aim of this section is threefold: 1) to prove that by knowing $\Gamma_{o}$ and $f_{c}$ it is possible to predict the echo response of the fault by means of the models proposed in sec. II; 2) that the peak value of the signal reflected by the fault does not represent the severity of the fault; 3 ) that the proposed simplified models, particularly (19) based on the derivative approximation, allows assessing the parameter $\left|\Gamma_{o}\right| / f_{c}$. All time-domain results involve baseband Gaussian pulses, for a varying bandwidth.

\section{A. Coaxial line}

The first set of tests involves the coaxial line depicted in Fig. 7. Two types of faults were considered: 1) a longitudinal cut along the line, exposing the internal portion of the cable, as in Fig. 7(a); 2) a simplified pigtail connection, as in Fig. 7(b), where the external conductor is reduced to a wire. This last configuration can be found in makeshift connections or could represent an advanced stage of deterioration in the original coaxial line. The main motivation for considering the pigtail connection is to observe the case of a relatively severe fault.

Five faults were considered: for the case in Fig. 7(a), three cut depths were studied, with $D=-0.2,0$ and $0.5 \mathrm{~mm}$, the pigtail configuration in Fig. 7(b), all for $w=10 \mathrm{~mm}$ and finally the case of a dent in the line, with $D=0 \mathrm{~mm}$ and $w=2 \mathrm{~mm}$.

The single-step reflectivity $\Gamma_{o}$, effective relative permittivity, critical and characteristic frequencies of the five faulty sections are summarized in Table I. Superficial cuts into the line involve a relatively weak single-step reflection, thus have a negligible impact on signal propagation, whereas the pigtail insert, displaying $\Gamma_{o}=0.45$, can still not be assimilated to a hard fault. Yet, the case of a cut with $D=-0.2 \mathrm{~mm}$, though corresponding to just $\Gamma_{o}=0.29$, cannot be regarded as a light modification, since in this case the internal conductor is almost severed. This last configuration is therefore interesting as an example of how the intuitive association between weak fault echoes and light wear in a transmission line is inexact. 


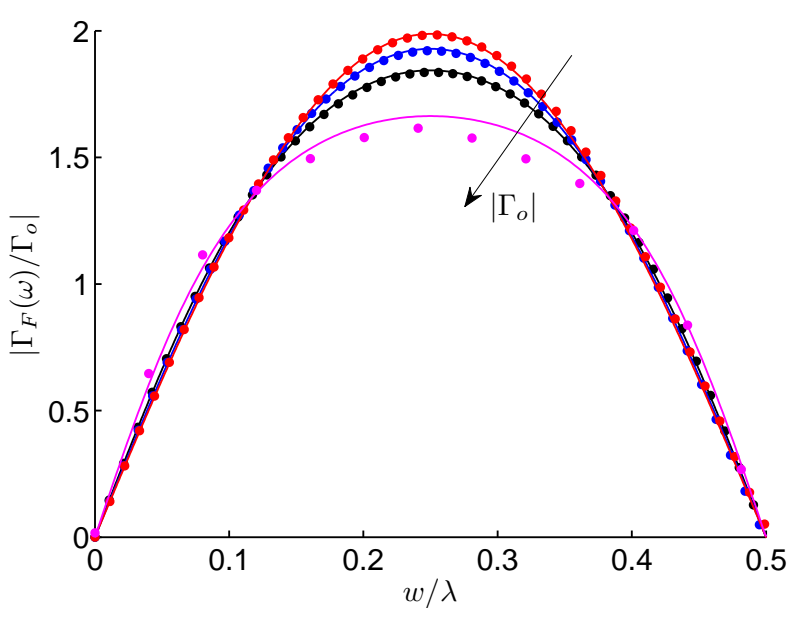

Fig. 8. Comparison between the numerical results for the configurations resumed in Table I, circles, and the prediction from (7), solid lines.

The validity of the exact echo-response model (7) is demonstrated in Fig. 8, where the data in Table I are fed to (7), with a good agreement between the numerical and theoretical results.

The time-domain responses of the echoes generated by the pigtail transition in Fig. 7(b) are shown in Fig. 9, for several bandwidths, characterized by $B_{o}$, defined in (14). Although the pigtail transition presents a normalized reflectivity $\Gamma_{F}(\omega) / \Gamma_{o}$ apparently closer to that expected for soft faults (Fig. 8) than for hard ones, it would be incorrect to rule out the use of the zero-pole approximation (9). The derivative approximation (16) is confirmed to hold for frequencies where $f / f_{c}$ is smaller than the values proposed in Fig. 6: for $\left|\Gamma_{o}\right|=0.45$, $f / f_{c} \lesssim 0.05 f_{o}$, i.e., from Table I, $f / f_{c} \lesssim 1.5 \mathrm{GHz}$. For wider bandwidths the zero-pole approximation keeps providing accurate results.

The cases (a) and (b) in Table II should be expected to provide the same estimates of $\Gamma_{o}$, assuming an a priori knowledge of $f_{c}$. Their minor disagreement is likely due to the non-negligible capacitive coupling between the two edges of the dent.

The accuracy of the proposed models suggests that they could be used not only as an analysis tool, but also the other way around, as a way of estimating the severity of the fault from its echoes. For the sake of simplicity, we will limit our analysis to the case of the derivative approximation, since

TABLE I

SiNGLE-STEP REFLECTION COEFFICIENT, EFFECTIVE RELATIVE DIELECTRIC PERMITTIVITY, CHARACTERISTIC FREQUENCY $f_{c}=c / w$, CRITICAL FREQUENCY $f_{o}=p / 2 \pi$ AND $\Gamma_{o} / f_{c}$, FOR SEVERAL FAULTY COAXIAL-LINE SECTIONS CONSIDERED IN THE NUMERICAL VALIDATION IN SEC. IV-A. DIMENSIONS $D$ AND $w$ ARE EXPRESSED IN MM.

\begin{tabular}{l|ccccc}
\hline \hline Configuration & $\Gamma_{o}$ & $\epsilon_{\mathrm{e}}$ & $\begin{array}{c}f_{c} \\
(\mathrm{GHz})\end{array}$ & $\begin{array}{c}f_{o} \\
(\mathrm{GHz})\end{array}$ & $\begin{array}{c}\Gamma_{o} / f_{c} \\
(\mathrm{ps})\end{array}$ \\
\hline cut, $D=0.5, w=10$ & 0.078 & 1.91 & 21.7 & 3.40 & 3.61 \\
cut, $D=0, w=10$ & 0.19 & 1.80 & 22.3 & 3.30 & 8.61 \\
dent, $D=0, w=2$ & 0.19 & 1.80 & 112 & 16.5 & 1.72 \\
cut, $D=-0.2, w=10$ & 0.29 & 1.77 & 22.5 & 3.02 & 12.9 \\
pigtail, $w=10$ & 0.45 & 1.00 & 29.9 & 3.16 & 15.1 \\
\hline \hline
\end{tabular}
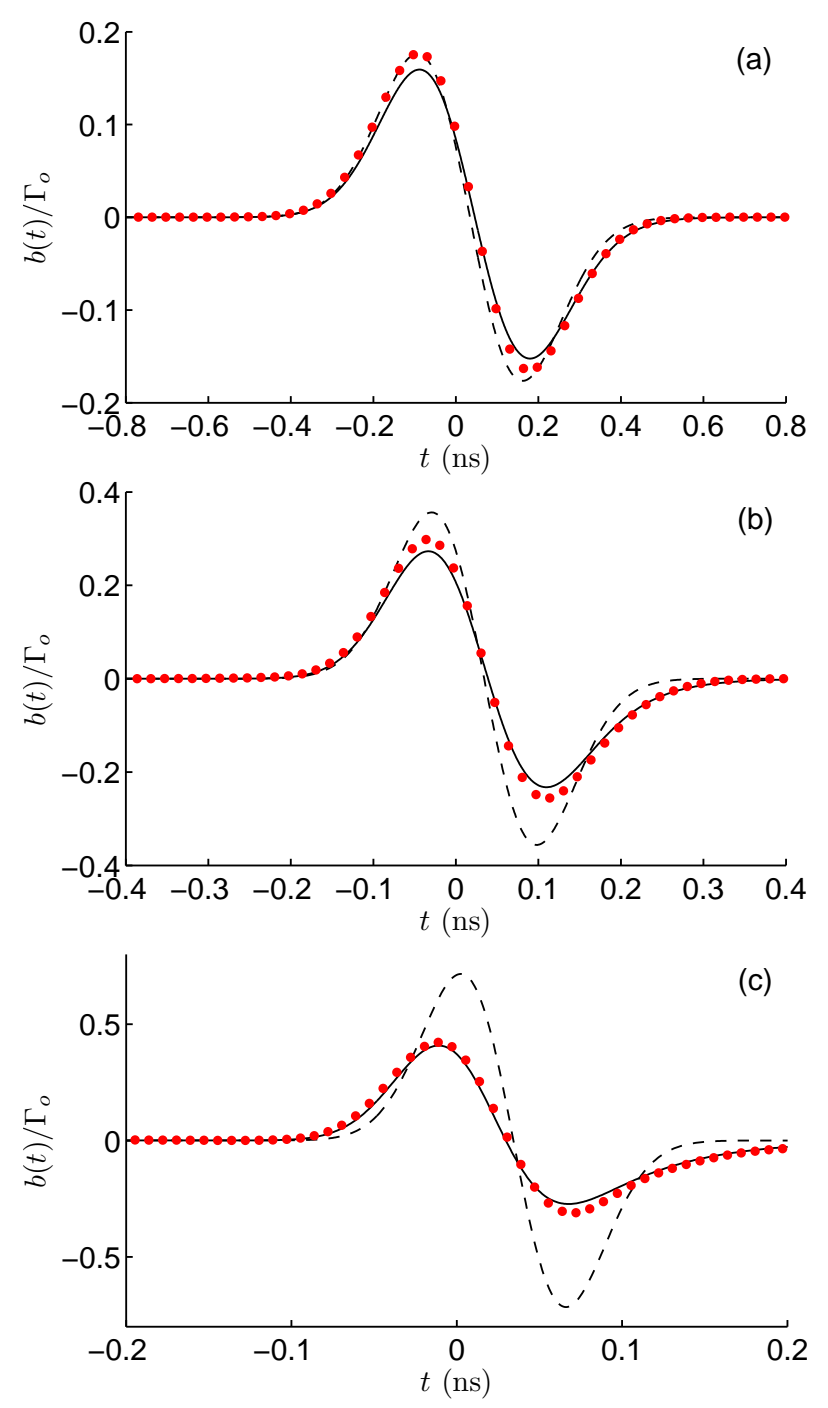

Fig. 9. Validation of the zero-pole model (9) and the derivative model (16), for the fault in Fig. 7(b) submitted to a Gaussian test signal with : (a) $B_{O}=$ $1.25 \mathrm{GHz}$, (b) $B_{o}=2.5 \mathrm{GHz}$ and(c) $B_{o}=5 \mathrm{GHz}$. Numerical results are shown as red circles, predictions from the zero-pole model as solid black lines and for the derivative model as dashed lines. The critical frequency is $f_{o}=3.16 \mathrm{GHz}$.

in practice the condition of electrically-short faults holds, in which case (18) is valid for Gaussian test signals. Clearly, other test signals can be considered.

This idea is validated by the results presented in Table II, for five faults and three bandwidths of the test signals. Starting from the peak intensity of the echoes, the severity of the fault is estimated back. The data in Table I serve as reference. From these results it is evident that the echo itself should not be interpreted as a measure of the severity of the fault. In particular, as discussed in sec. III, its peak amplitude can widely change of several orders of magnitude depending on the frequency bandwidth covered by the test signal.

More importantly, Table II confirms that the only parameter that can be derived under the derivative approximation is the ratio $\Gamma_{o} / f_{c}$. In order to translate it into a measure of the fault severity, the length $w$ of the fault needs to be known or assumed being contained in a given range of values. The 
TABLE II

RESULTS FOR A TDR IDENTIFICATION OF FAULTS IN A COAXIAL LINE, AS IN FIG. 7(A), WITH: (A) $D=0, w=10$ MM; (B) $D=0, w=2$ MM; (C) $D=0.5, w=10 \mathrm{MM}$; (D) $D=-0.2, w=10 \mathrm{MM}$; (E) PIGTAIL CONNECTION, $w=10$ MM. ESTIMATES DERIVED FROM THE ECHOES RESULTING FROM GAUSSIAN TEST SIGNALS WITH THREE VALUES OF BANDWIDTHS $B_{o}$, FOR : (1) $\Gamma_{o} / f_{c} ;$; 2 ) $\Gamma_{o}$, UNDER THE ASSUMPTION OF A PRIORI KNOWLEDGE OF THE FAULT EXTENSION $w$ AND THE PROPAGATION SPEED WITHIN THE FAULTY SECTION; (3) $\Gamma_{o}$, UNDER THE ASSUMPTION OF A PRIORI KNOWLEDGE OF THE FAULT EXTENSION $w$, ASSUMING THE PROPAGATION SPEED IN THE FAULTY SECTION TO BE EQUAL TO THAT OF THE NOMINAL TRANSMISSION LINE.

\begin{tabular}{c|ccccc}
\hline \hline Case & $\begin{array}{c}B_{o} \\
(\mathrm{GHz})\end{array}$ & $\max _{t}|b(t)|$ & $\begin{array}{c}\Gamma_{o} / f_{c}^{(1)} \\
(\mathrm{ps})\end{array}$ & $\Gamma_{o}^{(2)}$ & $\Gamma_{o}^{(3)}$ \\
\hline & 0.1 & $6.5710^{-3}$ & 8.62 & 0.192 & 0.178 \\
(a) & 0.3 & $2.0310^{-2}$ & 8.86 & 0.198 & 0.183 \\
& 1 & $6.6110^{-2}$ & 8.68 & 0.194 & 0.179 \\
\hline & 0.1 & $1.6610^{-3}$ & 2.17 & 0.242 & 0.225 \\
(b) & 0.3 & $4.6710^{-3}$ & 2.04 & 0.228 & 0.211 \\
& 1 & $1.4710^{-2}$ & 1.93 & 0.216 & 0.200 \\
\hline & 0.1 & $2.6510^{-3}$ & 3.48 & 0.00753 & 0.00718 \\
(c) & 0.3 & $8.2110^{-3}$ & 3.59 & 0.0778 & 0.0741 \\
& 1 & $2.6910^{-2}$ & 3.53 & 0.0765 & 0.0729 \\
\hline & 0.1 & $1.2910^{-2}$ & 13.5 & 0.303 & 0.279 \\
(d) & 0.3 & $3.1710^{-2}$ & 13.9 & 0.312 & 0.287 \\
& 1 & 0.102 & 13.4 & 0.302 & 0.278 \\
\hline & 0.1 & $1.7310^{-2}$ & 22.6 & 0.677 & 0.469 \\
(e) & 0.3 & $5.0610^{-2}$ & 22.1 & 0.662 & 0.458 \\
& 1 & 0.154 & 20.2 & 0.604 & 0.418 \\
\hline \hline
\end{tabular}

ratio $\Gamma_{o} / f_{c}$ is accurately estimated from the peak value of the reflected signals, within a few percent points. Assuming an a priori knowledge of $w$, or at least advancing typical guess values, also $\Gamma_{o}$ could be precisely extracted from the echoes, at least in principle. A further unknown is the speed of signal propagation along the faulty line. Taking it to be equal to the nominal value results in a source of systematic errors, even though of limited intensity.

The only significative disagreement appears for the case of the pigtail connection: having neglected the term $1 /\left(1-\Gamma_{o}^{2}\right)$ in the passage from (18) to (19), the severity assessed from the echoes is overestimated by this term, equal in this case to about $25 \%$. Using the value of $f_{c}$ in Table $\mathrm{I}$, the exact inversion of (18) provides the values $0.505,0.497$ and 0.470 for $\left|\Gamma_{o}\right|$, respectively for the three values of $B_{o}$ in Table II.

Unfortunately, the exact inversion of (18) is possible only if explicitly assuming an a priori knowledge of the characteristic frequency $f_{c}$ : since it is more realistic to regard it as unknown, it is safer, for a robust estimation, to apply (19).

\section{B. Two-wire line}

A further validation was carried out for the case of a twowire line, detailed in Fig. 10(a). Three typologies of faults were considered, with reference to Fig. 10: (a) a partial cut in the line; (b) the presence of a droplet of water over the line coating; c) a metallic slab partially inserted into the line coating. Cases (b) and (c) represent configurations where the line conductors are not yet affected: they stand for configurations where external actions can eventually lead to a fault. In fact, a water droplet can be any aqueous solution of corrosive liquids, which are a potential threat to the line integrity, while
TABLE III

SINGLE-STEP REFLECTION COEFFICIENT, EFFECTIVE RELATIVE DIELECTRIC PERMITTIVITY, CHARACTERISTIC FREQUENCY $f_{c}=c / w$, CRITICAL FREQUENCY $f_{o}=p / 2 \pi$ AND $\Gamma_{o} / f_{c}$, FOR THE FOUR FAULTY TWO-WIRE-LINE SECTIONS CONSIDERED IN THE NUMERICAL VALIDATION IN SEC. IV-B. DIMENSIONS $D$ AND $w$ ARE EXPRESSED IN MM.

\begin{tabular}{c|ccccc}
\hline \hline Configuration & $\Gamma_{o}$ & $\epsilon_{\mathrm{e}}$ & $\begin{array}{c}f_{c} \\
(\mathrm{GHz})\end{array}$ & $\begin{array}{c}f_{o} \\
(\mathrm{GHz})\end{array}$ & $\begin{array}{c}\left|\Gamma_{o}\right| / f_{c} \\
(\mathrm{ps})\end{array}$ \\
\hline cut, $D=0, w=10$ & 0.15 & 2.18 & 20.3 & 3.09 & 7.4 \\
cut, $D=0.5, w=10$ & 0.041 & 2.51 & 18.9 & 2.99 & 2.2 \\
water droplet, $w=5$ & -0.032 & 3.35 & 32.6 & 5.19 & 0.98 \\
slab, $D=0.6, w=5$ & -0.42 & 3.07 & 34.1 & 3.80 & 12.3 \\
\hline \hline
\end{tabular}

the metallic slab could cut through the remaining layer of insulating coating, resulting into a short circuit.

The characteristic data of four faults were computed and are shown Table III: they go from very light modifications in the propagation along the line to relatively severe ones, as for the case of the metallic slab with $D=0.6 \mathrm{~mm}$.

The echo responses of these four faults where computed for three bandwidths of a Gaussian test signal. From the peak intensity of the echoes we applied (19) in order to estimate the fault severity, as done in sec. IV-A. The results shown in Table IV support the validity of the proposed approach, with a good agreement between estimates from the echoes and the expected values obtained from numerical simulations, shown in Table III. The only disagreement appears, as was already the case for a coaxial line, for severe faults, here the case of the metallic slab: having neglected the term $1 /\left(1-\Gamma_{o}^{2}\right)$ in the passage from (18) to (19), the severity assessed from the echoes is overestimated by about $21 \%$. The disagreement is therefore explained by this missing term.

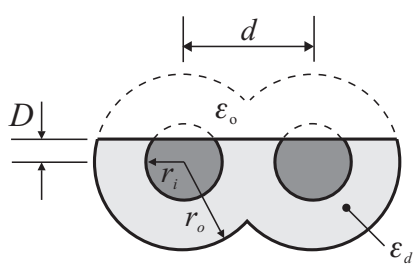

(a)

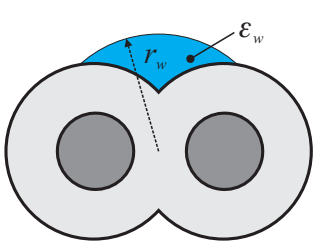

(b)

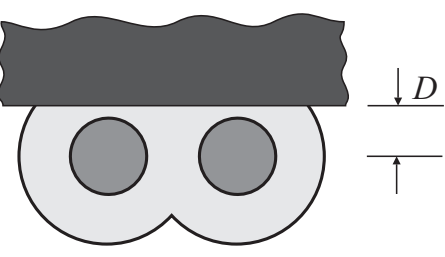

(c)
Fig. 10. Two-wire line with cylindrical conductors of radius $r_{i}=0.5 \mathrm{~mm}$, a dielectric coating of thickness $1 \mathrm{~mm}\left(r_{o}=1.5 \mathrm{~mm}\right)$ and relative dielectric constant $\epsilon_{d}=3.5$ (polyvinyl chloride, PVC). The distance between the conductors axis is $d=2 \mathrm{~mm}$. The pictures illustrate the three faults considered: (a) a partial cut in the line; (b) the presence of a water droplet of radius $r_{w}=2 \mathrm{~mm}$ over the two conductors and c) a metallic slab partially inserted into the line. 
TABLE IV

RESULTS FOR A TDR IDENTIFICATION OF FAULTS IN A TWO-WIRE LINE, AS IN FIg. 10, WITH: (A) CUT, WITH $D=0, w=10$ MM; (B) CUT, WITH $D=0.5, w=10 \mathrm{MM}$; (C) WATER DROPLET, WITH $w=5 \mathrm{MM}$; (D) METALLIC SLAB, 5 MM THICK, INSERTED INTO THE COATING AT $D=0.6$ MM FROM THE CENTER OF THE CONDUCTORS. ESTIMATES DERIVED FROM THE ECHOES RESULTING FROM GAUSSIAN TEST SIGNALS WITH THREE VALUES OF BANDWIDTHS $B_{o}$, FOR : (1) $\Gamma_{o} / f_{c}$; (2) $\Gamma_{o}$ UNDER THE ASSUMPTION OF A PRIORI KNOWLEDGE OF THE FAULT EXTENSION $w$ AND THE PROPAGATION SPEED WITHIN THE FAULTY SECTION; (3) $\Gamma_{o}$, UNDER THE ASSUMPTION OF A PRIORI KNOWLEDGE OF THE FAULT EXTENSION $w$, ASSUMING THE PROPAGATION SPEED IN THE FAULTY SECTION TO BE EQUAL TO THAT OF THE NOMINAL TRANSMISSION LINE.

\begin{tabular}{|c|c|c|c|c|c|}
\hline Case & $\begin{array}{c}B_{o} \\
(\mathrm{GHz})\end{array}$ & $\overline{\max _{t}|b(t)|}$ & $\begin{array}{c}\left|\Gamma_{o}\right| / f_{c}^{(1)} \\
(\mathrm{ps})\end{array}$ & $\overline{\Gamma_{o}^{(2)}}$ & $\Gamma_{o}^{(3)}$ \\
\hline \multirow{3}{*}{ (a) } & 0.1 & $5.7010^{-3}$ & 7.482 & 0.152 & 0.130 \\
\hline & 0.3 & $1.7310^{-2}$ & 7.564 & 0.154 & 0.132 \\
\hline & 1 & $5.6110^{-2}$ & 7.364 & 0.149 & 0.128 \\
\hline \multirow{3}{*}{ (b) } & 0.1 & $1.7510^{-3}$ & 2.289 & 0.045 & 0.040 \\
\hline & 0.3 & $5.0410^{-3}$ & 2.206 & 0.044 & 0.038 \\
\hline & 1 & $1.6110^{-2}$ & 2.118 & 0.042 & 0.037 \\
\hline \multirow{3}{*}{ (c) } & 0.1 & $0.7510^{-3}$ & 0.98 & -0.032 & -0.034 \\
\hline & 0.3 & $2.2210^{-3}$ & 0.97 & -0.032 & -0.039 \\
\hline & 1 & $7.2810^{-3}$ & 0.95 & -0.031 & -0.033 \\
\hline \multirow{3}{*}{ (d) } & 0.1 & $1.3110^{-2}$ & 17.2 & -0.56 & -0.60 \\
\hline & 0.3 & $3.8910^{-2}$ & 16.9 & -0.554 & -0.590 \\
\hline & 1 & $1.2410^{-1}$ & 16.3 & -0.530 & -0.565 \\
\hline
\end{tabular}

\section{CONCLUSIONS}

This paper has shown how the shape of the echo responses generated by a fault depend on its severity, yielding a mix of proportional and derivative contributions. Simple criteria for the identification of the fault response have been presented, by introducing the concept of a critical frequency of a fault, defined by means of a Padé approximant.

Practical implications of these results are that usual TDR techniques valid in the case of single-step discontinuities (hard faults) should be revised in order to account for the derivative nature of general faults. Potential systematic errors in the estimation of the fault position and intensity were highlighted in this respect, together with formulas allowing an accurate assessment of the severity of a fault, based on its characteristic frequency.

A major result is that severe faults a short step away from hard faults (e.g., almost severed lines) can respond with very weak echoes, if tested at frequencies well below their critical frequency. General conditions allowing a proper detection were then presented. The use of test signals with high-frequency content seems to be necessary, in order to ascertain whether an echo is generated by a severe fault or not, since echoes get stronger as the frequency increases. The demonstration that one needs an estimate of the fault extent $w$ represents a major issue for TDR fault detection in transmission lines.

While our analysis was based on the case of baseband Gaussian test signals, it can be extended to any other test signal in a straightforward manner. Furthermore, the case of correlationbased TDR techniques can also be considered, without any major difference in the validation and interpretation of the present analysis, by operating at the output of the receiving correlator.

\section{REFERENCES}

[1] L. Griffiths, R. Parakh, C. Furse, and B. Baker, "The invisible fray: A critical analysis of the use of reflectometry for fray location," IEEE Sensors Journal, vol. 6, no. 3, pp. 697-706, June 2006.

[2] B. M. Oliver, "Time domain reflectometry," Hewlett-Packard Journal, vol. 15 , no. 6 , pp. $1-7,1964$.

[3] C. Furse and R. Haupt, "Down to the wire," IEEE Spectrum, vol. 38, no. 2, pp. 34-39, 2001.

[4] C. Furse, Y. C. Chung, C. Lo, and P. Pendayala, "A critical comparison of reflectometry methods for location of wiring faults," Smart Structures and Systems, vol. 2, no. 1, pp. 25-46, 2006.

[5] G. D. Cormack, "Time-domain reflectometer measurement of random discontinuity effects on cable magnitude response," IEEE Transactions on Instrumentation and Measurement, vol. 21, no. 2, pp. 128 -135, May 1972.

[6] D. Ricker, Echo signal processing. Springer Netherlands, 2003, vol. 725 .

[7] C. Buccella, M. Feliziani, and G. Manzi, "Detection and localization of defects in shielded cables by time-domain measurements with UWB pulse injection and clean algorithm postprocessing," IEEE Transactions on Electromagnetic Compatibility, vol. 46, no. 4, pp. 597 - 605, November 2004.

[8] Y.-J. Shin, E. Powers, T.-S. Choe, C.-Y. Hong, E.-S. Song, J.-G. Yook, and J. B. Park, "Application of time-frequency domain reflectometry for detection and localization of a fault on a coaxial cable," IEEE Transactions on Instrumentation and Measurement, vol. 54, no. 6, pp. 2493 - 2500, December 2005.

[9] M. Kafal, A. Cozza, and L. Pichon, "Locating multiple soft faults in wire networks using an alternative dort implementation," IEEE Transactions on Instrumentation and Measurement, vol. 65, no. 2, pp. 399-406, Feb 2016.

[10] - "Locating faults with high resolution using single-frequency trmusic processing," IEEE Transactions on Instrumentation and Measurement, in press.

[11] K. Kurokawa, "Power waves and the scattering matrix," IEEE Transactions on Microwave Theory and Techniques, vol. 13, no. 2, pp. $194-$ 202, march 1965.

[12] R. Collin, Foundations for microwave engineering. John Wiley \& Sons, 2007.

[13] G. Baker Jr., "Essentials of Padé approximants," New York, 1975.

[14] C. Glader, G. Högnäs, P. Mäkilä, and H. Toivonen, "Approximation of delay systems - a case study," International Journal of Control, vol. 53, no. 2, pp. 369-390, 1991.

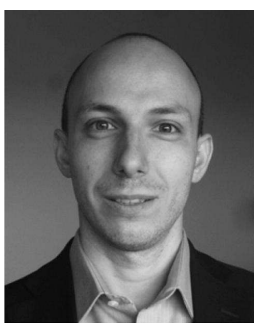

Andrea Cozza (S'02 - M'05 - SM'12) received the Laurea degree (summa cum laude) in electronic engineering from Politecnico di Torino, Turin, Italy, in 2001, and the Ph.D. degree in electronic engineering jointly from Politecnico di Torino and the University of Lille, France, in 2005

In 2007, he joined the Département de Recherche en Électromagnétisme, SUPELEC, Gif sur Yvette, France, where since 2013 he is full professor. He is a reviewer for several scientific journals, including those of IET and IEEE. His current research interests include reverberation chambers, statistical electromagnetics, wave propagation through complex media and applications of time reversal to electromagnetics.

Dr. Cozza was awarded the 2012 Prix Coron-Thévenet from the Académie des Sciences, France. 


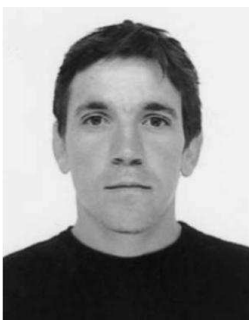

Lionel Pichon was born in Romorantin, France, in 1961. He received the Dip. Eng. degree from Ecole Supérieure dŠIngénieurs en Electronique et Electrotechnique, Noisy Le Grand, France, in 1984.

In 1985, he joined the Laboratoire de Génie Electrique de Paris, Gif sur Yvette, France, where he received the $\mathrm{Ph} . \mathrm{D}$. degree in electrical engineering in 1989. He got a position at the Centre National de la Recherche Scientifique (CNRS), Paris, France, in

1989. He is currently Directeur de Recherche at the CNRS. His research interests include computational electromagnetics for wave propagation, scattering, electromagnetic compatibility, and nondestructive testing. 